\title{
Physical activity and prostate cancer: a systematic review
}

\begin{abstract}
Numerous studies confirmed that planned exercise therapy is a possible adjunct strategy connected with significant improvements in symptom-related results including exercise tolerance as well as several cancer patients-reported progress such as improvement in quality of life, and physical functioning during conventional adjuvant therapy. The aim of this review was to evaluate the association between physical activity and prostate cancer. Apart from lung cancer, prostate cancer is highly prevalent among men. We searched for original articles, systematic reviews, and meta-analyses that reported on exercise-mediated changes in the prostatic tumour risk and progression from 1980 to 2018. The following electronic databases was used: PubMed, Science Direct, Medline, Sports Discus, Web of Science, Google Scholar and Cochrane database. 85 studies written in English were included in this review. Patient's cardio-metabolic profile, type of exercise, specific workloads, frequency, duration, intensity and safety precautions are factors to consider when scheduling an exercise program. Regular participation in physical activity is important in the prevention of prostate cancer and it is associated with positive treatment outcomes in patients undergoing Androgen deprivation therapy. Physical Activity may affect prostate cancer progression by reducing insulin resistance, decreasing bioavailable Insulin-like growth factor 1 (IGFI), increasing adiponectin levels and circulating levels of insulin. Interleukin 6 (IL-6) promotes cell proliferation and inhibits apoptosis of prostate cancer cells in vitro. Physical activity is associated with lower circulating IL-6. Based on the information examined in this study, physical activity may be an effective nonpharmacological means in the treatment of prostate cancer.
\end{abstract}

Keywords: physical activity, exercise, prostate tumor, cancer prevention
Volume 7 Issue 5 - 2019

\author{
Abdalla Ali Deb,' Chidiebere Emmanuel \\ Okechukwu, ${ }^{2}$ Shady Emara, ${ }^{3}$ Sami A Abbas ${ }^{4}$ \\ 'Locum Consultant Urologist NHS- UK \\ ${ }^{2}$ Physical activity and health promotion, Department of \\ Biomedicine and prevention, Faculty of Medicine and Surgery, \\ University of Rome Tor vergata. Rome, Italy \\ ${ }^{3}$ Clinical Fellow in Urology, Western General Hospital UK \\ ${ }^{4}$ Consultant Urologist, National Institute of Urology and \\ Nephrology Cairo; Egypt
}

Correspondence: Abdalla Ali Deb, Locum Consultant Urologist NHS- UK, Email drabdodeeb@hotmail.com

Received: October 06, 2019 | Published: October 30, 2019

\section{Introduction}

For the past 2 decades, improved research and clinical attention has focused on the effectiveness of exercise therapy as an adjunct approach for the treatment of cancer. ${ }^{1}$ Randomized trials validate that lanned exercise therapy is a feasible adjunct tactic connected with significant improvements in symptom-related outcomes including exercise tolerance as well as multiple patient-reported treatment progress such as improvement in quality of life and physical functioning equally during conventional adjuvant therapy. ${ }^{2}$ In this review, we outline relevant studies related to exercise-mediated changes in the prostatic tumour risk and progression from 1980 to 2018. Prostate cancer is the second most prevalent form of cancer diagnosed among men after lung cancer. ${ }^{3}$ Androgen deprivation therapy (ADT) is applied in the treatment of patients in the advanced stage of prostate cancer. The side effects of ADT include erectile dysfunction, ${ }^{4}$ increase fat mass, ${ }^{5}$ and reduction in muscle strength. ${ }^{6}$ Combined resistance and aerobic exercise training program was effective in reversing muscle loss in men undergoing ADT due to prostate cancer. ${ }^{7}$ Pelvic floor/sphincter training is effective in plummeting incontinence in patients with prostate cancer. ${ }^{89}$ The molecular mechanisms behind the positive effects of exercise training programs in addition to ADT has not been well understood, however there was a decrease in inflammation due to low nuclear factor- $\mathrm{KB}$ activation in $\mathrm{LNCaP}$ cells incubated with post-exercise serum ${ }^{10}$ and suppressed growth and increased apoptosis of $\mathrm{LNCaP}$ cells incubated with post-exercise serum. ${ }^{11}$ Exercise alters p53, p21 and caspase activities resulting in tumour growth inhibition, tumour apoptosis, tumour suppression and suppressed metastasis. ${ }^{12},{ }^{13}$ Physical activity was effective in reducing prostate carcinogenesis in transgenic model. ${ }^{14}$ The demonstration of possible mechanisms whereby exercise alters the progression of carcinogenesis might strengthen the clinical outcome of cancer treatment.

Exercise could also result to reductions in obesity and oxidative stress and a modulation of immune responses in prostate cancer patients. ${ }^{15}$ Exercise causes reductions in circulating levels of testosterone and insulin-like growth factors ${ }^{16,17}$ therefore reducing the development and spread of neoplastic cells. Apart from exercise, testosterone levels are controlled by diet, ${ }^{18}$ and this may contribute to variances in exercise response among various populations. ${ }^{19}$ The aim of this review was to evaluate the association between physical activity and prostate cancer. In this review, we outlined relevant studies concerned about exercise-mediated changes in prostatic growth and progression from 1980 to 2018. These mediated changes are linked to the role of consistent physical activity in improving the quality of life, physical fitness and averting the progression of prostate cancer among individuals diagnosed of prostate cancer.

\section{Methods}

\section{Search strategy}

We searched for studies that reported on exercise-mediated changes in the prostatic tumour risk and progression from 1980 to 2018. The following electronic databases was used: PubMed, Science 
Direct, Medline, Sports Discus, Web of Science, Google Scholar and Cochrane database. The following search strategy was modified for the various databases and search engines: Prostate cancer, exercise, physical activity, cancer, prostate tumour, cancer prevention, and cancer adjunct therapy. We assessed Full articles and extracted relevant data. We used the MeSH system to extract relevant research studies indexed in PubMed.

\section{Types of studies}

Original articles, systematic reviews, and meta-analyses.

\section{Inclusion and exclusion criteria}

We selected precise articles that described physical activity and prostate growth, exercise therapy in support of the clinical treatment of prostate cancer and exercise prescription for prostate cancer patients. The selected articles were all written in English. Articles that were not precise, uncertain and with doubtful experimental procedure were excluded. 85 studies were included in this review.

\section{Data extraction and management}

We designated all trials retrieved from the databases. We reviewed for relevance based on physical activity and prostate cancer progression, cancer prevention, exercise therapy for prostate cancer patients undergoing ADT and radiotherapy. We retrieved full-text copies of all the articles recognised as hypothetically relevant in this review. Data was reported in a narrative and concise manner. The selection process was elucidated in a Preferred Reporting Items for Systematic Reviews and Meta-analyses (PRISMA) flow diagram (Figure 1).

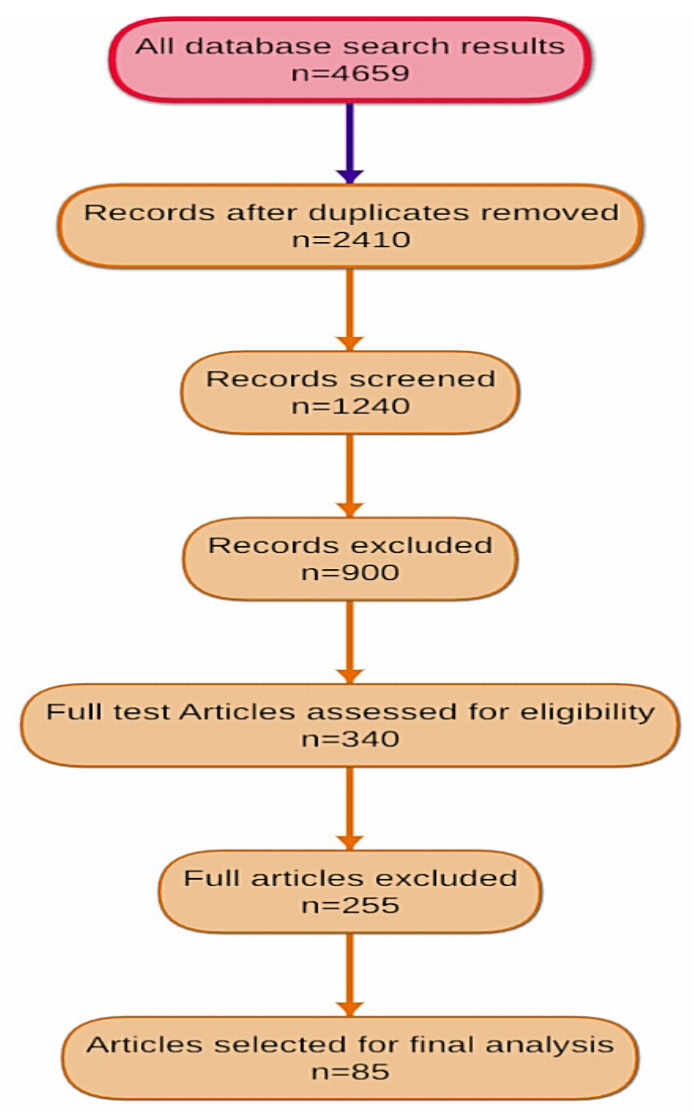

Figure I Preferred Reporting Items for Systematic Reviews and Meta-analyses (PRISMA) flow diagram.

The effectiveness of exercise prescription and dosing for patients diagnosed with prostate cancer

Patient's cardio-metabolic profile, type of exercise, specific workloads, frequency, duration, intensity and safety precautions are factors to consider when planning an exercise program. Regular involvement in physical activity is vital in the prevention of prostate cancer and it is connected with positive treatment results in patients undergoing ADT. ${ }^{20}$ Patients diagnosed with prostate cancer should accumulate at least; 150 min per week of moderate-intensity aerobic exercise or $75 \mathrm{~min}$ per week of vigorous aerobic exercise, or both, this is in accordance with the reference exercise strategies prescribed by the American College of Sports Medicine (ACSM) for cancer patients ${ }^{21,22}$ Cardiovascular diseases are the leading cause of death in men with prostate cancer ${ }^{23}$ and exercise performed according to the standard prescription improves cardiovascular fitness and averts cardiovascular deaths. ${ }^{24}$ Galvao et al. ${ }^{25}$ found a significant increase in muscle strength in prostate cancer patients allocated to the group that took part in chest press, a significant increase in muscle stamina was detected in the group that participated in chest press, there was significant increase in Muscle thickness at the quadriceps position. There was substantial development in general cardiovascular fitness, 
flexibility, muscle strength, quality of life, and drastic reduction in fatigue among prostate cancer patients undertaking radiotherapy after undergoing an 8-week cardiovascular exercise-training program ${ }^{26}$ A randomised controlled trial conducted by Segal et al. ${ }^{27}$ in which Prostate cancer patients completed resistance exercise training 3 times per week, demonstrated global progresses in quality of life, muscular strength, body fat ratio decrease and lessening in fatigue in the group that partook in resistance exercise and are undertaking radiotherapy, both aerobic and resistance training was found to lessen fatigue. A personalized exercise-training program planned and prescribed according to patients' cardio-metabolic and functional capacity might be more effective in the treatment of prostate cancer. ${ }^{28}$ The ideal approach and dosage of exercise for patients diagnosed of prostate cancer has not been established.

\section{Selected studies on physical activity and prostate cancer}

According to the World Health Organization (WHO), physical activity is any physical movement produced by skeletal muscles that involves energy expenditure. ${ }^{29}$ Based on experimental and clinical studies, physical activity averts the progression of prostate cancer by changing molecular mechanisms resulting to the suppression of tumour growth. In tabulating available epidemiological facts, we categorized them by type of study (cross-sectional, prospective cohort, or case-control) and by findings (an adverse effect, no clear response, a positive trend), and a statistically significant decrease of risk in the more active individuals. There have been many cross-sectional or cohort studies relating occupation to the risk of prostate cancer (Table 1). Leisure-time physical activity have also been linked with the risk of developing prostate cancer based on cohort studies (Table 2). Four retrospective and cohort studies of sport involvement and fitness realization was critically analysed (Table 3 )., two of these information found no benefit from sport involvement, ${ }^{70}$ but Wannamethee et al.$^{64}$ noted a considered outcome on the reported frequency of sport participation, However, the fourth report observed that a beneficial effect was connected with a high-attained level of aerobic fitness. ${ }^{71}$

Table I Occupational activity and the risk of developing prostate cancer

\begin{tabular}{|c|c|c|c|c|}
\hline Author & Sample & Activity measure & Outcome & Comments \\
\hline \multicolumn{5}{|c|}{$\begin{array}{l}\text { Cohort studies } \\
\text { Negative findings }\end{array}$} \\
\hline Hartman et al. ${ }^{30 a}$ & $\begin{array}{l}29,133 \text { male smokers, } \\
\text { aged } 50-69 \text { years } \\
\text { followed for an average } \\
\text { of } 6.1 \text { years; } 317 \text { incident } \\
\text { cases of PC }\end{array}$ & $\begin{array}{l}\text { Self-report, sedentary job } \\
\text { vs. occupational walkers } \\
\text { vs. walkers/lifters vs. heavy } \\
\text { laborers }\end{array}$ & $\begin{array}{l}\text { RR I. } 0,0.6,0.8, \text { I. } 2 \text { (ns) of prostate } \\
\text { cancer as stated by Finnish cancer } \\
\text { registry }\end{array}$ & $\begin{array}{l}\text { Attuned for age, } \\
\text { urban living, smoking, } \\
\text { benign prostate } \\
\text { hyperplasia }\end{array}$ \\
\hline Johnsen et al. ${ }^{3 \mathrm{la}}$ & $\begin{array}{l}\text { I27,923 men, aged } \\
20-97 \text { years, followed } \\
\text { for } 8.5 \text { years; } 2458 \text { cases } \\
\text { of } P C\end{array}$ & $\begin{array}{l}\text { Two classifications by } \\
\text { interview or questionnaire: } \\
\text { sitting, standing, or manual } \\
\text { work; inactive, moderately } \\
\text { inactive, moderately active, } \\
\text { active }\end{array}$ & $\begin{array}{l}\text { Occupational activity not significantly } \\
\text { related to PC (OR manual } 0.90 \text { (0.77- } \\
\mathrm{I} .04), \mathrm{P}=0.15 \text { for trend) }\end{array}$ & $\begin{array}{l}\text { Attuned for leisure } \\
\text { activity, height, } \\
\text { weight, marital } \\
\text { status, education }\end{array}$ \\
\hline Nielsen et al. ${ }^{32 a}$ & $\begin{array}{l}22,895 \text { Norwegian men } \\
\text { aged } 40 \text { to }>80 \text { years } \\
\text { followed for } 9.3 \text { years; } \\
644 \text { cases of } P C\end{array}$ & $\begin{array}{l}\text { Modest binary classification } \\
\text { (high vs. low level of } \\
\text { occupational activity) }\end{array}$ & $\begin{array}{l}\text { No effect of occupational activity } \\
\text { ((high RR I.04 (0.82-I.32); augmented } \\
\text { risk in high vs. low education, RR I.50 } \\
(I . I I-2.19)\end{array}$ & $\begin{array}{l}\text { Age-adjusted } \\
\text { relative risks }\end{array}$ \\
\hline Putnam et al. ${ }^{33 a}$ & $\begin{array}{l}\text { I0I cases of PC were } \\
\text { conveyed to State } \\
\text { register office, I } 572 \\
\text { initially cancer-free } \\
\text { men aged } 40-86 \text { years, } \\
\text { followed for } 4 \text { years }\end{array}$ & $\begin{array}{l}\text { Very energetic, ascetically } \\
\text { active, or inactive at work, } \\
\text { based on work-related codes }\end{array}$ & $\begin{array}{l}\text { PC unrelated to occupational activity (RR } \\
\text { for very active } 1.0(0.6-1.8))\end{array}$ & Adjusted for age \\
\hline Zeegers et al. ${ }^{34 a}$ & $\begin{array}{l}58,279 \text { men aged } \\
55-69 \text { years, } 1386 \text { cases } \\
\text { of } P C \text { over } 9.3 \text { years of } \\
\text { follow-up }\end{array}$ & $\begin{array}{l}\text { Work-related activities } \\
\text { (estimated energy } \\
\text { expenditure, sitting time for } \\
\text { longest held and for most } \\
\text { recent job) }\end{array}$ & $\begin{array}{l}\text { RRs longest held: }>12 \text { vs. }<8 \mathrm{~kJ} / \mathrm{min} 0.9 \mathrm{I} \\
(0.70-\mathrm{I} . \mathrm{I} 8) \text { Sitting }<2 \mathrm{vs} .>6 \mathrm{~h} / \text { dayl. } 16 \\
(0.9 \mathrm{I}-\mathrm{I} .47)\end{array}$ & $\begin{array}{l}\text { Attuned for age, } \\
\text { alcohol drinking, } \\
\text { BMI, energy intake, } \\
\text { family history, } \\
\text { education }\end{array}$ \\
\hline
\end{tabular}


Table Continued

\begin{tabular}{|c|c|c|c|c|}
\hline Author & Sample & Activity measure & Outcome & Comments \\
\hline \multicolumn{5}{|l|}{ Positive trend } \\
\hline Grotta et al. ${ }^{35 a}$ & $\begin{array}{l}13,109 \text { Swedish men, } \\
\text { initially aged } 55 \text { years, } \\
\text { surveyed for } 13 \text { years; } \\
904 \text { cases of PC }\end{array}$ & $\begin{array}{l}\text { Low vs medium vs. high level } \\
\text { of work-related activity }\end{array}$ & $\begin{array}{l}\text { HR for high activity } 0.8 \mathrm{I}(0.6 \mathrm{I}-\mathrm{I} .07, \mathrm{~ns}) \text {, } \\
\text { medium } 0.96(0.77-\mathrm{I} .20)\end{array}$ & $\begin{array}{l}\text { Attuned for age, } \\
\text { education, smoking, } \\
\text { BMI, alcohol } \\
\text { consumption, } \\
\text { diabetes mellitus }\end{array}$ \\
\hline $\begin{array}{l}\text { Hrafnkelsdóttir } \\
\text { et al. }{ }^{36 a}\end{array}$ & $\begin{array}{l}24 \text {-year follow-up } \\
\text { of } 822 \text { I Icelandic } \\
\text { men initially aged } \\
33-79 \text { years; } 1052 \text { cases } \\
\text { of PC }\end{array}$ & $\begin{array}{l}\text { Work includes mostly sitting } \\
\text { vs standing vs on the move }\end{array}$ & $\begin{array}{l}\text { HR I.0, } 0.97 \text { (0.80-I.I7), 0.9I (0.79- } \\
\text { I.06), ns }\end{array}$ & $\begin{array}{l}\text { Attuned for age, } \\
\text { height, BMI, diabetes, } \\
\text { family history, } \\
\text { education, medical } \\
\text { check-ups }\end{array}$ \\
\hline
\end{tabular}

Significant positive findings

\begin{tabular}{|c|c|c|}
\hline Clarke et al. ${ }^{37 a}$ & $\begin{array}{l}5377 \text { men firstly, aged } \\
25-75 \text { years surveyed } \\
\text { for I7-2I years; } 20 \text { I } \\
\text { cases of PC }\end{array}$ & Very active vs. sedentary \\
\hline Norman et al. ${ }^{38}$ & $\begin{array}{l}3 \text { cohorts containing } \\
43,836,28,702 \text {, and } \\
19,670 \text { cases of PC }\end{array}$ & $\begin{array}{l}\text { Work-related designations } \\
\text { (inactive, medium, to very } \\
\text { high level of activity) }\end{array}$ \\
\hline
\end{tabular}

45,887 men aged 45-

Orsini et al $39 \mathrm{a} \quad 79$ years, surveyed for 8 years; 2735 incident cases of PC

$\begin{array}{ll}\text { Parent et al. }{ }^{40 a} & \begin{array}{l}449 \text { incident cases of } \\ \text { PC; aged } 59 \text { years }\end{array} \\ & \begin{array}{l}\text { 60, } 194 \text { men initially aged } \\ \text { Vidarsdottir et }\end{array} \\ {\text { al. }{ }^{41}}^{204} \text { years followed } \\ \text { for } 23 \text { years }\end{array}$

\section{Case-control studies}

\section{Adverse findings}

\section{Negative findings}

$\begin{array}{ll}\text { Doolan et al. }^{42} & \begin{array}{l}\text { 1436 cases of PC aged } \\ 39-70 \text { years, I } 349 \\ \text { coordinated controls }\end{array} \\ \text { Hosseini et al. }{ }^{43} \quad \begin{array}{l}\text { I } 37 \text { cases of PC, I } 37 \\ \text { locality controls, men } \\ <70 \text { to }>80 \text { years }\end{array}\end{array}$

258 cases of PC, 47I

Lacey et al. ${ }^{44 a} \quad$ age-matched controls, aged 50-94 years

760 PC cases aged

Sass-Kortak et al. $^{45}$
50-84 years, 1632

telephone book controls
Finnish occupation background, physical capacity classified by tertiles

Walking to work ( $<10$ vs. $>10 \mathrm{~h} /$ week), intensity of work (sedentary/moderately active vs. highly active)

Sedentary, moderate, or high work-related energy expenditures at ages 20-29 years, $40-49$ years, or 12 years ago

Quartiles of lifetime workrelated activity
RR for inactive 1.75 ( $1.12-2.67), p=0.05$ for trend (outcome larger in African Americans)

RR for sedentary vs. high/very high groups I.II, I.I0, and I.II ( $p=0.000$ I for all three groups)

4 categories of occupation (mostly sitting vs. heavy manual)

High vs. medium vs. low lifetime occupational activity, metabolic equivalents (METs)

Educational level (basic, medium, high)
$R R=0.72(0.57-0.90) ; p=0.007$ for trend; effects smaller for advanced and fatal cancers

OR intermediate $0.64(0.4 I-0.98)$, high $0.54(0.31-0.95)$

SIR basic $=0.92(0.84-0.99)$, academic $=I .17(1.05-1.30)$
Attuned for age, education, ethnicity, family history

Attuned for age, year of follow-up, area of residence

Attuned for leisure activity, age, smoking, alcohol consumption, education, diet, energy intake, waist/ hip ratio, diabetes mellitus

Data attuned for age, sex, education, ethnicity, smoking, BMI, outdoor activities

Greater diagnosis in highly educated group
OR highest tertile workload I.I5 (0.95I.40), ns

OR 0.7 (0.4-I.2) for longer walk (ns),

$\mathrm{OR}=6.7(\mathrm{I} .3-35 . \mathrm{I})$ for highly active work Multivariate adjusted $(p=0.02)$

RR I.I (0.7-I.7), I.3 (0.8-I.9), 0.9 (0.5I.8) of high vs. sedentary group

Active vs. least active workers OR I.33 ( $1.02-1.74), p=0.18$ for trend)
Attuned for age, family history, economic resources

Attuned for age, marital status, education, BMI, energy intake, waist/ hip ratio

Attuned for age, family history, sunlight exposure 
Table Continued

\begin{tabular}{|c|c|c|c|c|}
\hline Author & Sample & Activity measure & Outcome & Comments \\
\hline \multicolumn{5}{|l|}{ No effect } \\
\hline $\begin{array}{l}\text { Friedenreich et } \\
\text { al. }^{46 a}\end{array}$ & $\begin{array}{l}988 \text { cases of PC, } \\
\text { histologically confirmed, } \\
1063 \text { population } \\
\text { controls }\end{array}$ & $\begin{array}{l}\text { Energy expenditure < } 74.2 \text { vs. } \\
>161.9 \mathrm{MET} \text { h/week }\end{array}$ & OR $0.90(0.60-1.22)$, ns & $\begin{array}{l}\text { Attuned for age, } \\
\text { region, education, } \\
\text { BMl, waist/hip } \\
\text { ratio, energy intake, } \\
\text { alcohol drinking, } \\
\text { family and medical } \\
\text { history }\end{array}$ \\
\hline
\end{tabular}

\section{Positive trend}

$\begin{array}{ll}\text { Lagiou et al. }{ }^{47} & \begin{array}{l}320 \text { histologically } \\ \text { confirmed cases pf P } \\ \text { aged }<60 \text { to }>80 \text { yea } \\ 246 \text { hospital controls }\end{array} \\ & \\ & \text { I449 incident cases } \\ \text { Wiklund et al. }{ }^{48 a} \text { in men aged } & \text { 35-79 years, I I I8 } \\ \text { population controls }\end{array}$

Low, medium, high level of job-related activity

METs h/day of lifetime workrelated activity, $<|| .8,<\mid 4.8$, $<19.8,>19.8$
OR low $=1.0$, medium $=0.95(0.49-1.84)$, high $=0.69(0.40-1.22)$, ns

OR I.0, 0.81 (0.65-I.08), 0.87 (0.66I.I5) 0.84 (0.6I-I.I5), ns
Attuned for age and education

Attuned for age, region, education, BMI, alcohol consumption, family history, diabetes mellitus, energy intake

\section{Significant positive findings}

\begin{tabular}{|c|c|}
\hline Bairati et al. ${ }^{49}$ & $\begin{array}{l}64 \text { cases of PC, } 5456 \\
\text { cases of benign prostate } \\
\text { hyperplasia aged } \\
>45 \text { years }\end{array}$ \\
\hline $\begin{array}{l}\text { Krishnadasan } \\
\text { et al. }{ }^{50}\end{array}$ & $\begin{array}{l}362 \text { cases of PC, I } 805 \\
\text { matched controls; age } \\
\text { not specified }\end{array}$ \\
\hline
\end{tabular}

I 294 incident cases of Pierotti et al. ${ }^{51 a} \quad$ PC aged $<75$ years and I45I hospital controls

176 cases of $P C$ in

Strom et al. ${ }^{52 a}$ Mexican Americans, 176 controls, age $\sim 62$ years

1623 histologically

$\begin{array}{ll}\text { Villeneuve et } & \text { confirmed cases of PC, } \\ \text { al. }{ }^{53} & \text { I623 controls, aged } \\ & 50-74 \text { years }\end{array}$

(a) inactive job or light work; (b) $0,1-49 \%,>50 \%$ of profession spent in inactive or light work

Low vs. moderate vs. high work-related energy outflow

3-level categorization of work-related activity at ages 12, 15-19, 30-39, and 50-59 years

None/low vs. moderate/high energy demands of labour

4-level classification of work (sitting to energetic)
OR high $0.63(0.40-1.00)$, moderate 0.96 (0.7-I.3), $p=0.06$ for trend)

(a) OR 2.0 (I.I-3.6); I.0, I.7 (0.8-3.2),

OR $2.8(1.3-6.0)(p=0.007$ for trend $)$

OR age 12 years $=0.84(0.67-1.06)$, age I $5-19$ years $=0.94(0.75-1.17)$, age $30-39$ years $=0.78(0.63-0.97)$, age $50-59$ years $=0.75(0.6 I-0.93)$

Reduced risk in active (OR 0.46, 0.280.77), $p=0.003$ )

Significant advantage from strenuous activity in teens or early $20 \mathrm{~s}$, (OR light 0.8 (0.5-I.3), moderate 0.8 (0.5-I.2), strenuous $0.6,0.4-0.9)$, ns for $30 \mathrm{~s}(0.7)$, $50 \mathrm{~s},(0.8)$ or 2 years before interview (0.9)
Attuned for age, education, total energy intake, smoking, use of vitamin supplements

Attuned for matching variables, pay, trichloroethylene exposure

Attuned for age, test centre, education, Sex, BMI, total energy intake, smoking, alcohol consumption, family history

Attuned for age, education, screening, exposure to agricultural chemicals

Attuned for age, area of residence, smoking, alcohol consumption, BMI, diet, income, family history

HR, hazard ratio; MET, metabolic equivalent, ns not significant; OR, odds ratio; PC, prostate cancer; PMR, proportionate mortality ratio; RR, relative risk or rate ratio; $\mathrm{SES}$, socioeconomic status; SIR, standardized incidence ratio 
Table 2 Leisure-time physical activity and the risk of developing prostate cancer

\begin{tabular}{|c|c|c|c|c|}
\hline Author & Sample & Activity measure & Findings & Comments \\
\hline \multicolumn{5}{|c|}{$\begin{array}{l}\text { Cohort studies } \\
\text { Adverse trend } \\
\text { Negative findings }\end{array}$} \\
\hline Crespo et al. ${ }^{54}$ & $\begin{array}{l}9824 \text { men initially aged } \\
35-79 \text { years followed for } \\
\text { death }\end{array}$ & $\begin{array}{l}\text { Framingham index } \\
\text { (quartiles) }\end{array}$ & $\begin{array}{l}\text { No association between physical } \\
\text { activity and prostate deaths }\end{array}$ & $\begin{array}{l}\text { Adjusted for age, education, urban } \\
\text { residence, smoking, BMI }\end{array}$ \\
\hline $\begin{array}{l}\text { Giovannucci } \\
\text { et al. }{ }^{55}\end{array}$ & $\begin{array}{l}47,452 \text { health experts } \\
\text { initially aged } 40-75 \text { years, } \\
\text { followed for } 8 \text { years; I } 362 \\
\text { cases of PC }\end{array}$ & $\begin{array}{l}\text { Leisure activity, I vs. } \\
\text { 46.8 MET h/week }\end{array}$ & $\begin{array}{l}\text { No significant association to PC } \\
\text { except idea of less metastatic } \\
\text { activity and lower Gleeson score } \\
\text { with vigorous intensity exercise }\end{array}$ & $\begin{array}{l}\text { Attuned for age, vasectomy, } \\
\text { diabetes mellitus, smoking, energy } \\
\text { intake, diet }\end{array}$ \\
\hline Grotta et al. ${ }^{35 b}$ & $\begin{array}{l}\text { I3, I09 Swedish men, } \\
\text { initially aged } 55 \text { years, } \\
\text { tracked for } 13 \text { years; } 904 \\
\text { cases of PC }\end{array}$ & $\begin{array}{l}\text { Low vs. high leisure } \\
\text { activity }\end{array}$ & $\begin{array}{l}\text { HR } 0.93(0.76-\mathrm{I} .14, \mathrm{~ns}) \text { for } \\
\text { incident PC if high physical activity }\end{array}$ & $\begin{array}{l}\text { Attuned for age, education, } \\
\text { smoking, BMI, alcohol drinking, } \\
\text { diabetes mellitus }\end{array}$ \\
\hline Johnsen et al. ${ }^{3 l a}$ & $\begin{array}{l}\text { I } 27,923 \text { men, median } \\
\text { initial age } 6 \text { I years, } \\
\text { followed for } 8.5 \text { years; } \\
2458 \text { cases of PC }\end{array}$ & $\begin{array}{l}\text { Quartiles of leisure } \\
\text { activity }(<25 \text { to }>7 \text { I } \\
\text { MET h/week) }\end{array}$ & $\begin{array}{l}\text { Leisure activity unrelated to } \\
\text { incident PC }\end{array}$ & $\begin{array}{l}\text { Attuned for work-related activity, } \\
\text { height, weight, marital status, } \\
\text { education }\end{array}$ \\
\hline Lee et al. ${ }^{56}$ & $\begin{array}{l}8922 \text { Harvard alumni, } \\
\text { mean age } 67 \text { years; } 439 \\
\text { developed PC during } \\
5 \text { years of follow-up }\end{array}$ & $\begin{array}{l}\text { Physical activity } \\
\text { questionnaire } \\
\text { completed twice, } \\
\text { weekly energy } \\
\text { expenditure quartiles } \\
\text { (<4.2 MJ to }>12.6 \mathrm{MJ})\end{array}$ & $\begin{array}{l}\text { PC disparate to total volume of } \\
\text { physical activity or weekly volume } \\
\text { of vigorous physical activity }\end{array}$ & $\begin{array}{l}\text { Accustomed for age, BMI, smoking, } \\
\text { alcohol consumption, family } \\
\text { history }\end{array}$ \\
\hline Littman et al. ${ }^{57}$ & $\begin{array}{l}34,757 \text { men initially aged } \\
50-76 \text { years; } 583 \text { cases } \\
\text { of PC }\end{array}$ & $\begin{array}{l}\text { MET h/week, walking } \\
\text { pace, stair climbing, } \\
\text { high-intensity activity, } \\
\text { activity at earlier ages }\end{array}$ & $\begin{array}{l}\text { No association with } P C \text { except } \\
\text { in sub-group aged }>65 \text { years with } \\
\text { normal body mass }\end{array}$ & $\begin{array}{l}\text { Attuned for family history, BMI, } \\
\text { income }\end{array}$ \\
\hline Liu et al. ${ }^{58}$ & $\begin{array}{l}982 \text { cases of } P C \text { in } 22,07 \text { I } \\
\text { physicians aged } 40- \\
84 \text { years over II years of } \\
\text { follow-up }\end{array}$ & $\begin{array}{l}\text { Exercise sufficient to } \\
\text { cause a sweat }<1 / \text { week. } \\
\text { vs. }>5 / \text { weeks }\end{array}$ & $\begin{array}{l}\text { No relationship of frequent } \\
\text { activity to incidence of PC }\end{array}$ & $\begin{array}{l}\text { Adjusted for smoking, alcohol } \\
\text { drinking, height, diabetes mellitus, } \\
\text { high cholesterol, hypertension, use } \\
\text { of multi-vitamins }\end{array}$ \\
\hline Parent et al. ${ }^{40 \mathrm{~b}}$ & $\begin{array}{l}449 \text { incident cases of } \\
\text { PC among } 3730 \text { cancer } \\
\text { patients }\end{array}$ & $\begin{array}{l}\text { participation in sports } \\
\text { and outdoor activities } \\
\text { (never or not often vs. } \\
\text { often) }\end{array}$ & $\begin{array}{l}\text { No significant consequence on } \\
\text { risk of PC }\end{array}$ & $\begin{array}{l}\text { Covariates age, SES, education, } \\
\text { ethnicity, smoking, BMI }\end{array}$ \\
\hline Platz et al. ${ }^{59}$ & $\begin{array}{l}46,786 \text { health } \\
\text { professionals, initially } \\
\text { aged } 40-75 \text { years; } 2896 \\
\text { incident cases of PC over } \\
\text { I4 years }\end{array}$ & $\begin{array}{l}\text { Vigorous leisure activity } \\
<3,>3 \mathrm{MET} \text { h/week }\end{array}$ & No relationship to $\mathrm{PC}$ & $\begin{array}{l}\text { Attuned for age, family history, } \\
\text { BMI, diabetes mellitus, smoking, } \\
\text { diet }\end{array}$ \\
\hline Putnam et al. ${ }^{33 b}$ & $\begin{array}{l}\text { I0I cases of PC in } 1572 \\
\text { initially cancer-free } \\
\text { men, originally aged } \\
40-64 \text { years, followed for } \\
4 \text { years }\end{array}$ & $\begin{array}{l}\text { Very active, moderately } \\
\text { active, inactive }\end{array}$ & $\begin{array}{l}\text { Risk of PC unrelated to leisure } \\
\text { activity }\end{array}$ & Attuned for total energy intake \\
\hline
\end{tabular}


Table Continued

\begin{tabular}{llll}
\hline Author Sample & Activity measure & Findings
\end{tabular}

\section{Positive trends}

\begin{tabular}{|c|c|}
\hline Clarke et al. ${ }^{37 b}$ & $\begin{array}{l}5377 \text { men, aged }<50 \text { to } \\
>70 \text { years, followed for } \\
\text { I7-2I years; } 20 \text { I cases of } \\
\text { incident or fatal PC }\end{array}$ \\
\hline $\begin{array}{l}\text { Giovannucci } \\
\text { et al. }{ }^{60}\end{array}$ & $\begin{array}{l}47,620 \text { health specialists, } \\
\text { initially aged } 40-75 \text { years, } \\
\text { I } 4 \text { years of follow-up; } \\
2892 \text { incident cases of PC } \\
\text { (482 advanced, } 280 \text { fatal) }\end{array}$ \\
\hline $\begin{array}{l}\text { Hrafnkelsdóttir } \\
\text { et al. }{ }^{36 \mathrm{~b}}\end{array}$ & $\begin{array}{l}24 \text { years of follow-up of } \\
822 \text { Icelandic men initially } \\
\text { aged } 33-79 \text { years; } 1052 \\
\text { cases of PC }\end{array}$ \\
\hline
\end{tabular}

cases of PC

\section{2,895 Norwegian}

Nielsen et al ${ }^{32 \mathrm{~b}}$ men, initially aged 40 to $>80$ years, followed for 9.3 years; 644 cases of $P C$

293,902 men initially aged Moore et al. ${ }^{61} \quad 50-71$ years followed for up to 8.2 years; 17,872 cases of PC

29, I I0 Norwegian men, initially mean age

Nilsen et al. ${ }^{62} \quad 52$ years, followed for 7 years; 957 incident cases of PC

72, 174 men, initial mean age 64 years; 5503 incident cases of PC over 9 years

58,279 men initially aged

Zeegers et al. ${ }^{34 b} \quad 55-69$ years; I 386 cases of $\mathrm{PC}$ over 9.3 years
Much vs. moderate vs. little or none

Vigorous physical activity, 0 vs. $>29$ MET h/week

Regular physical activity from age of 20 years vs. sedentary

High vs. low leisure activity

\section{Exercise at starting} point and in adolescence (never/ rarely to $>5$ times/ week)

Activity score based on frequency, intensity and duration of activity (low vs. high)

MET h/week (<0.7-35) at age 40 years

Cycling/walking ( $\mathrm{min} /$ day), gardening (h/week)
RR much I.00, moderate I.I0 (0.75-I.6I), inactive I.I7 (0.80I.72), ns

No relationship for all subjects; if $>65$ years, OR for advanced cancer $0.33(0.17-0.62)$

HR 0.93 (0.83-I.07) for all PC in active individuals, $0.82(0.63-1.06)$ for advanced cancers

RR $0.80(0.62-1.03)$

RR of total cases $0.97(0.91-$ I.03), $p=0.03$ for inclination supporting frequent activity during adolescence, but no relationships to exercise habits at baseline

Connected to total cancer cases $(R R=0.86)$, but for advanced cancer $R R=0.64$ (0.43$0.95), p=0.02$ for inverse trend

No significant effect (but active have fewer hostile tumours, RR $0.69(0.52-0.92), p=0.06$ for trend

Gardening unrelated to PC; biking/walking < 10 vs. $>60 \mathrm{~min} / \mathrm{d}$, RR 0.85 (0.69-I.05), ns
Attuned for age, education, ethnicity, family history

Age, BMI, smoking, height, family history, diabetes mellitus, ethnicity, non-vigorous activity, energy intake and diet

Accustomed for age, height, BMI, diabetes, family history, education, medical check-ups

Multivariate adjusted

Accustomed for age, marital status, education, smoking, medical history, BMI, waist circumference, family history, diet and supplements

Attuned for age, marital status, education, BMI, smoking, alcohol consumption

Accustomed for age, ethnicity, BMI, weight change, energy intake, diet and vitamin use, diabetes mellitus, family and medical history

Accustomed for age, alcohol drinking, BMI, energy intake, family history, gardening, sport participation

\section{Significant positive findings}

$\begin{array}{ll}\begin{array}{l}\text { Hartman et } \\ \text { al. }^{30 a}\end{array} & \begin{array}{l}29,133 \text { male smokers, } \\ \text { initially aged 50-69 years, } \\ \text { followed for up to } \\ 9 \text { years; } 317 \text { cases of PC }\end{array} \\ & 45,887 \text { men, initially aged } \\ & 45-79 \text { years, followed for } \\ \text { Orsini et al. }{ }^{39 b} & 8 \text { years; } 2735 \text { incident } \\ & \text { cases of PC }\end{array}$

Wannamethee Potential study of 7588 et al. ${ }^{64 a}$ men aged 40-59 years;

120 incident cases of PC
Inactive vs, moderate/ heavy leisure activity in working men

Walking or cycling, 5 categories (hardly ever to $>60 \mathrm{~min} /$ day)

6-level classification of leisure activity from none to vigorous
RR 0.7 (0.46-0.94) favoring active leisure

$R R=0.86(0.76-0.98), p=0.028$ for trend; effects greater for advanced $(R R=0.74)$ and fatal $(R R=0.72)$ cancers

Advantage from vigorous activity, OR $0.25(0.06-0.99, \mathrm{p}=0.06$ for trend
Attuned for age, urban living, smoking, benign hyperplasia

Attuned for work-related activity, age, smoking, alcohol drinking, education, diet, energy intake, waist/hip ratio, diabetes mellitus

Attuned for age, smoking, alcohol consumption, BMI, social class 
Table Continued

\begin{tabular}{|c|c|c|c|c|}
\hline Author & Sample & Activity measure & Findings & Comments \\
\hline \multicolumn{5}{|c|}{ Case-control studies } \\
\hline Chen et al. ${ }^{65}$ & $\begin{array}{l}237 \text { cases of } P C, 48 I \\
\text { controls aged }>50 \text { years }\end{array}$ & $\begin{array}{l}\text { Mainly a dietary study: } \\
\text { 4-level categorization of } \\
\text { physical activity }\end{array}$ & $\begin{array}{l}\text { Adverse effect of high vs. } \\
\text { moderate exercise: OR I.84 } \\
(1.01-3.34)\end{array}$ & $\begin{array}{l}\text { Multivariate analysis (age, BMI, } \\
\text { income, marriage, dietary } \\
\text { variables) }\end{array}$ \\
\hline $\begin{array}{l}\text { Wiklund et } \\
\text { al. }{ }^{48 \mathrm{~b}}\end{array}$ & $\begin{array}{l}\text { I } 449 \text { incident cases of } \\
\text { PC, I I I } 8 \text { population } \\
\text { controls, mean age } \\
67-68 \text { years }\end{array}$ & $\begin{array}{l}\text { MET } h / \text { day lifetime } \\
\text { recreational activity, } \\
<7.4 \text { to }>13.5\end{array}$ & $\begin{array}{l}\text { OR }<7.4=1.0,<10.2=1.33(1.00- \\
I .78),<13.5=1.43(1.07-1.9 \mid) \\
>13.5=1.56(1.16-2.10), p=0.006 \\
\text { for adverse effect of active leisure }\end{array}$ & $\begin{array}{l}\text { Attuned for age, region, education, } \\
\text { BMI, alcohol consumption, family } \\
\text { history, diabetes mellitus, energy } \\
\text { intake }\end{array}$ \\
\hline
\end{tabular}

\section{No clear effect} matched controls, aged 50-94 years

1294 incident cases of PC

Pierotti et al. ${ }^{5 \mathrm{la}}$ aged $<75$ years and $\mid 45$ I hospital controls

416 incident cases

Sanderson et al. ${ }^{66}$

Strom et al. ${ }^{52 b}$

176 cases of PC in Mexican Americans, 176 controls, age $\sim 62$ years

I 623 histologically

Villeneuve et al. ${ }^{53 \mathrm{~b}}$ confirmed cases of PC, 1623 controls, age 50-74 years
Tertiles of moderate/ vigorous or all physical activity at age 20-29 years, age 4049 years, and 12 years ago

3-level classification of physical activity at ages 12, 15-19, 30-39, and 50-59 years

Tertiles of strenuous and of moderate physical activity (h/ week)

Leisure activity $(<1 /$ week vs. > I/week)

5-level classification $(<1 /$ month to $>5$ /week)
No association to PC

No effect on risk of PC at any age

No relationship to $P C$ in either African American or Caucasian men

No effect on risk of PC

No clear relationship to $\mathrm{PC}$
Attuned for age, marital status, education, BMI, energy intake, waist/hip ratio

Accustomed for age, test center, education, SES, BMI, total energy intake, smoking, alcohol drinking, family history

Adjusted for age, geographic region, family history

\section{Positive trends}

Friedenreich et al. ${ }^{46 \mathrm{~b}}$
988 incident cases of PC, 1063 population controls, mean age 67 years
$<78.5$ vs. $>25$. I MET h/ week
OR I.00, $0.80(0.61-1.04), p=0.06$ for trend
Accustomed for age, region, education, BMI, waist/hip ratio, energy intake, alcohol drinking, family and medical history 
Table Continued

\begin{tabular}{|c|c|c|c|c|}
\hline Author & Sample & Activity measure & Findings & Comments \\
\hline \multicolumn{5}{|c|}{ Significant positive findings } \\
\hline $\begin{array}{l}\text { Darlington et } \\
\text { al. }{ }^{67}\end{array}$ & $\begin{array}{l}752 \text { cases from Ontario } \\
\text { cancer registry aged } \\
50-84 \text { years, telephone } \\
\text { listing controls }\end{array}$ & $\begin{array}{l}\text { Strenuous activity mid- } \\
\text { teens, early } 30 \text { s, early } \\
50 \text { s (yes/no) }\end{array}$ & $\begin{array}{l}\text { OR for strenuous activity in } \\
\text { teens }=1.0(0.8-1.2) \text {, early } \\
30 \mathrm{~s}=0.9(0.7-1.0) \text {, early } 50 \mathrm{~s}=0.8 \\
(0.6-0.9) \text {. }\end{array}$ & $\begin{array}{l}\text { Attuned for age, education, BMI, } \\
\text { family history, occupation }\end{array}$ \\
\hline Jian et al. ${ }^{68}$ & $\begin{array}{l}\text { I } 30 \text { histologically } \\
\text { confirmed cases of PC, } \\
274 \text { controls, aged }<65 \text { to } \\
>75 \text { years }\end{array}$ & $\begin{array}{l}\text { Reported MET hours } \\
\text { of moderate and total } \\
\text { activity }(<40 \text { vs }>120 \text {; } \\
<44 \text { vs. }>135)\end{array}$ & $\begin{array}{l}\text { OR moderate activity }<40=1.0, \\
<80=0.47(0.22-1.02),<120=0.46 \\
(0.21-0.99),>120=0.20(0.07- \\
0.62), p=0.015, \text { Total activity } \\
<44=1.0,<90=0.42(0.18-0.99), \\
<135=0.36(0.16-0.86), \\
>135=0.39(0.15-0.99), p=0.50 \\
\text { for trend) }\end{array}$ & $\begin{array}{l}\text { Accustomed for age, area of } \\
\text { residence, education, salary, marital } \\
\text { status, number of children, years } \\
\text { in labour force, family history, BMI, } \\
\text { energy intake }\end{array}$ \\
\hline Yu et al. ${ }^{69}$ & $\begin{array}{l}\text { I I } 62 \text { cases of PC; } \\
3 \text { I } 24 \text { matched hospital } \\
\text { controls, age }<45 \text { to } \\
>75 \text { years }\end{array}$ & $\begin{array}{l}\text { Leisure activity (active, } \\
\text { moderate, or seldom) }\end{array}$ & $\begin{array}{l}\text { Risk higher in sedentary } \\
(\text { OR seldom }=.3(I .0-1.6) \\
\text { moderate }=1.1(0.9-1.3) \\
\text { active }=1.0(p=0.03)\end{array}$ & Attuned for age \\
\hline
\end{tabular}

BMI, body mass index; HR hazard ratio; MET, metabolic equivalent; ns non-significant; NHANES, National Health and Nutrition Examination Survey; OR, odds ratio; $P C$, prostate cancer; $R R$, relative risk; SES, socioeconomic status

Table 3 Sports involvement, attained aerobic fitness, and risk of prostate cancer

\begin{tabular}{|c|c|c|c|c|}
\hline Author & Sample & Activity measure & Findings & Comments \\
\hline \multicolumn{5}{|l|}{ Cohort studies } \\
\hline Merrill et al. ${ }^{70}$ & $\begin{array}{l}\text { PSA levels of } \\
536 \text { partakers } \\
\text { in senior citizen } \\
\text { games aged } \\
>50 \text { years }\end{array}$ & Years active $>3$ times/week & $\begin{array}{l}\text { Total physical activity unrelated to PSA } \\
\text { levels }\end{array}$ & Attuned for age \\
\hline Zeegers et al. ${ }^{34 c}$ & $\begin{array}{l}58,279 \text { men aged } \\
55-69 \text { years; } \\
\text { I } 386 \text { cases of } P C \\
\text { over } 9.3 \text { years }\end{array}$ & $\begin{array}{l}\text { Sport involvement (never/ever; frequency; } \\
\text { duration, year) }\end{array}$ & Sport involvement unrelated to PC & $\begin{array}{l}\text { Attuned for age, } \\
\text { alcohol consumption, } \\
\text { BMI, energy intake, } \\
\text { family history, } \\
\text { education }\end{array}$ \\
\hline \multicolumn{5}{|c|}{ Case-control studies } \\
\hline $\begin{array}{l}\text { Hållmarker et } \\
\text { al. } .^{72}\end{array}$ & $\begin{array}{l}185,412 \\
\text { partakers in } \\
\text { Vasaloppet ski } \\
\text { contest and } \\
184,617 \text { non- } \\
\text { participants }\end{array}$ & I827 vs. 1435 cases $P C$ & $\begin{array}{l}\text { HR I.22 (I.I3-I.30) backing non- } \\
\text { participants }\end{array}$ & $\begin{array}{l}\text { Non-participants } \\
\text { matched for age, sex, } \\
\text { county of residence }\end{array}$ \\
\hline Galvão ${ }^{73}$ & $\begin{array}{l}57 \text { prostate } \\
\text { cancer patients, } \\
\text { aged } 70.0 \pm 8.4 \\
\text { year }\end{array}$ & $\begin{array}{l}\text { Randomized to multimodal supervised } \\
\text { aerobic, resistance, and flexibility } \\
\text { exercises undertaken thrice weekly } \\
\text { (exercises }(E X) ; n=28 \text { ) or usual care (care } \\
\text { controls (CON);n=29) for } 3 \text { months. }\end{array}$ & $\begin{array}{l}\text { A significant difference between } \\
\text { groups for self-reported physical } \\
\text { functioning ( } 3.2 \text { points; } 95 \% \text { confidence } \\
\text { interval, } 0.4-6.0 \text { points; } P=0.028) \\
\text { and lower body muscle strength }(6.6 \\
\mathrm{kg} ; 95 \% \text { confidence interval, } 0.6- \\
\mathrm{I} 2.7 ; \mathrm{P}=0.033) \text { at } 3 \text { months favouring } \\
\mathrm{EX} .\end{array}$ & With bone metastases \\
\hline
\end{tabular}

BMI, body mass index; HR, hazard ratio; ns non-significant; PC, prostate cancer; PSA, prostate serum antigen; RR, relative risk; SES, socioeconomic status; EX, exercises 


\section{Possible biological mechanisms linking exercise and prostate cancer}

EL Richman et al. ${ }^{74}$ Observed that walking pace was related to a lesser risk of prostate cancer independently of walking period, they stated that men who walked 3 or more hours/week at a brisk pace had a 57\% lower risk of prostate cancer. Physical Activity may affect prostate cancer progression by reducing insulin resistance, decreasing bioavailable Insulin-like growth factor 1 (IGFI), increasing adiponectin levels and circulating levels of insulin ${ }^{75}$ (Figure 2). Interleukin 6 (IL-6) promotes cell proliferation and inhibits apoptosis of prostate cancer cells in vitro ${ }^{76}$ however, physical activity is associated with lower circulating IL-6. ${ }^{77}$ There is an increase in tumor blood flow in animal models, during intense endurance exercise, due to the change in vasculature, thus causing an upsurge in tumor perfusion and oxygenation and in turn decreases the propensity for metastasis. ${ }^{78}$ Regulation of tumor vasculature might be a likely biological mechanism through which exercise prevents prostate cancer progression. Elevated serum levels of IGF-1, leptin and insulin are related to higher risk of prostate cancer progression. ${ }^{79,80} \mathrm{LNCaP}$ cells cultured with post exercise serum, expresses reduction in cell proliferation and increased apoptosis. ${ }^{81}$

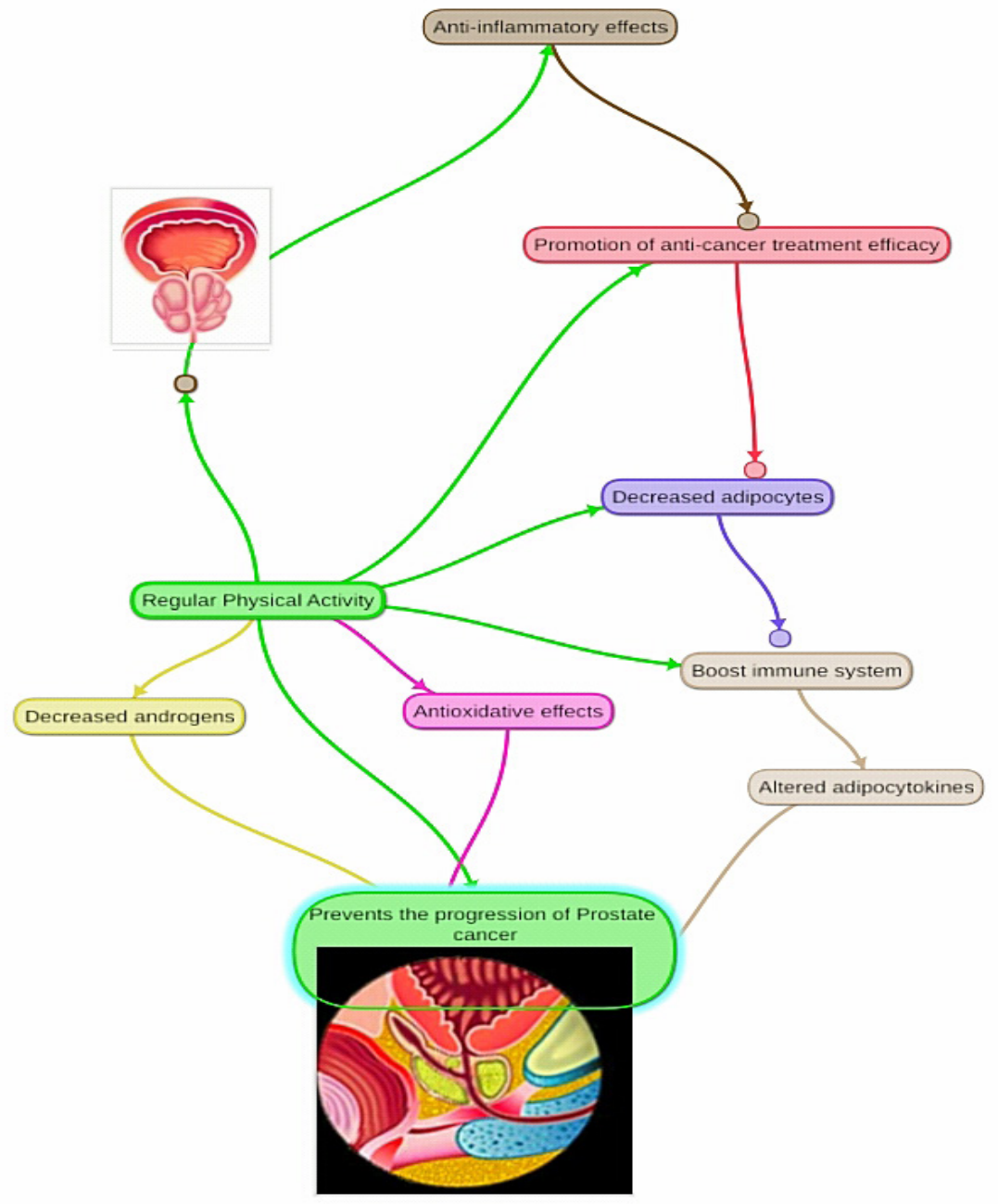

Figure 2 Possible Biological mechanisms linking Physical activity and Prostate Cancer.

Exercise lessens the side effects associated with androgen deprivation treatment for Prostate cancer. ${ }^{82}$ Aerobic exercise exerts cardio-protective effects in animal-subjected to ADT. ${ }^{83}$ Exercise results to an upsurge in the production of sex hormone-binding globulin (SHBG), dropping testosterone levels ${ }^{84}$ Exercise boost antioxidantenzyme repair mechanisms and drops lipid peroxidation levels, in turn plummeting free radicals and minimizing oxidative stress ${ }^{85}$ Regular physical activity combined with lifestyle adjustment and exogenous natural supplements in addition to the pharmacological, surgical and radiotherapeutic treatment of prostate cancer may enable clinicians to attain the anticipated treatment goal.

\section{Conclusion}

Based on the information obtained and analysed in this study, a practical exercise and activity goal personalized for prostate cancer patients to increase their chances of survival would be a 
multidimensional tactic to decrease central adipose tissue deposition and to lessen circulating levels of inflammation, insulin, and unfavourable sex hormones. Further studies aimed to assess the most effective exercise therapy and actual dose, personalised according to patients' clinical status for averting prostate cancer progression are vital in order to develop a comprehensive prevention and treatment goal for prostate cancer. Physical activity levels, ranging from walking to more intense activities and exercise routines, offers unique benefits. data from Randomized trials continue to accumulate regarding the positive effects of exercise on treatment outcomes for men with prostate cancer and the implementation of exercise during and after treatment for prostate cancer ought to be part of the standard of care and treatment goal. The radiation oncologists are provided with exceptional chance to restate healthy lifestyle methods and modifications owing to the extensive time spent with patients on a weekly basis during treatment. Prostate cancer treatment is one of the lengthiest treatment regimens, and the oncologist is given several opportunities to recommend and aid to implement exercise and lifestyle changes.

\section{Acknowledgments}

None.

\section{Funding details}

None.

\section{Conflicts of interest}

The author declares there is no conflict of interest.

\section{References}

1. Friedenreich CM, Woolcott CG, McTiernan A, et al. Alberta physical activity and breast cancer prevention trial: Sex hormone changes in a year-long exercise intervention among postmenopausal women. $J$ Clin Oncol. 2010;28(9):1458-1466.

2. Scott JM, Zabor EC, Schwitzer E, et al. Efficacy of exercise therapy on cardiorespiratory fitness in patients with cancer: A systematic review and meta-analysis. J Clin Oncol. 2018;36(22):2297-2305.

3. Quinn M, Babb P. Patterns and trends in prostate cancer incidence, survival, prevalence and mortality, I: international comparisons. BJU Int. 2002;90(2):62-173.

4. Zelefsky MJ, Eid JF. Elucidating the etiology of erectile dysfunction after definitive therapy for prostate cancer. Int J Radiat Oncol Biol Phys. 1998;40(1):129-133.

5. Smith MR, Finkelstein JS, McGovern FJ, et al. Changes in body composition during androgen deprivation therapy for prostate cancer. $J$ Clin Endocrinol Metab. 2002;87(2):599-603.

6. Taylor LG, Canfield SE, Du XL. Review of major adverse effects of androgen deprivation therapy in men with prostate cancer. Cancer. 2009; 115(11):2388-2399.

7. Galvao DA, Taaffe DR, Spry N, et al. Combined resistance and aerobic exercise program reverses muscle loss in men undergoing androgen suppression therapy for prostate cancer without bone metastases: a randomized controlled trial. J Clin Oncol. 2010;28(2):340-347.

8. Filocamo MT, Li Marzi V, Del Popolo G, et al. Effectiveness of early pelvic floor rehabilitation treatment for post-prostatectomy incontinence. Eur Urol. 2005; 48(5):734-738.
9. Overgard M, Angelsen A, Lydersen S, et al. Does physiotherapist-guided pelvic floor muscle training reduce urinary incontinence after radical prostatectomy? A randomized controlled trial. Eur Urol. 2008;54(2):438448.

10. Soliman S, Aronson WJ, Barnard RJ. Analyzing serum-stimulated prostate cancer cell lines after low-fat, high-fiber diet and exercise intervention. Evid Based Complement Altern Med. 2011;2011:529053.

11. Rundqvist H, Augsten M, Stromberg A, et al. Effect of acute exercise on prostate cancer cell growth. PLoS One. 2013;8(7): e67579.

12. Leung PS, Aronson WJ, Ngo TH, et al. Exercise alters the IGF axis in vivo and increases 553 protein in prostate tumor cells in vitro. $J$ Appl Physiol. 2004;96(2):450-454.

13. Barnard RJ, Leung PS, Aronson WJ, et al. A mechanism to explain how regular exercise might reduce the risk for clinical prostate cancer. Eur $J$ Cancer Prev. 2007;16(5):415-421.

14. Esser KA, Harpole CE, Prins GS, et al. Physical activity reduces prostate carcinogenesis in a transgenic model. Prostate. 2009;69(13):1372-1377.

15. Shephard RJ, Shek PN. Associations between physical activity and susceptibility to cancer. Sports Medicine. 1998;26(5):293-315.

16. Wekesa A, Harrison M, Watson RW. Physical activity and its mechanistic effects on prostate cancer. Prostate Cancer Prostatic Dis. 2015;18(3):197207.

17. Wheeler GD, Wall SR, Belcastro AN, et al. Reduced serum testosterone and prolactin levels in male distance runners. JAMA. 1984; 252(4):514 516.

18. Hämäläinen EK, Adlercreutz H, Puska P, Pietinen P. Decrease of serum total and free testosterone during a low-fat high-fibre diet. J Steroid Biochem. 1983;18(3):369-370.

19. Sung JF, Lin RS, Pu YS, et al. Risk factors for prostate carcinoma in taiwan: A case-control study in a chinese population. Cancer. 1999;86(3):484-491.

20. Davies NJ, Batehup L, Thomas R. The role of diet and physical activity in breast, colorectal and prostate cancer survivorship: a review of the literature. Br J Cancer. 2011;105(1):S52-S73.

21. Kushi LH, Doyle C, McCullough M, et al. American Cancer Society guidelines on nutrition and physical activity for cancer prevention: reducing the risk of cancer with healthy food choices and physical activity. CA Cancer J Clin. 2012;62(1):30-67.

22. Schmitz KH, Courneya KS, Matthews C, et al. American College of Sports Medicine roundtable on exercise guidelines for cancer survivors. Med Sci Sports Exerc. 2010;42(7):1409-1426.

23. Ketchandji M, Kuo YF, Shahinian VB, et al. Cause of death in older men after the diagnosis of prostate cancer. J Am Geriatr Soc. 2009;57(1):24 30 .

24. Pate RR, Pratt MP, Blair SN, et al. Physical activity and public health: a recommendation from the Centers for Disease Control and Prevention and the American College of Sports Medicine. JAMA. 1995;273(5):402-407.

25. Galvao DA, Nosaka K, Taaffe DR, et al. Resistance training and reduction of treatment side effects in prostate cancer patients. Med Sci Sports Exerc. 2006;38(12):2045-2052.

26. Monga U, Garber SL, Thornby J, et al. Exercise prevents fatigue and improves quality of life in prostate cancer patients undergoing radiotherapy. Arch Phys Med Rehabil. 2007;88(11):1416-1422.

27. Segal RJ, Reid RD, Courneya KS, et al. Resistance exercise in men receiving androgen deprivation therapy for prostate cancer. J Clin Oncol. 2003;21(9):1653-1659. 
28. Demark WW, Clipp EC, Lipkus IM, et al. Main outcomes of the FRESH START trial: a sequentially tailored, diet and exercise mailed print intervention among breast and prostate cancer survivors. J Clin Oncol. 2007;25(19):2709-2718.

29. World Health Organization (WHO). Global Strategy on Diet, Physical Activity and Health. 2004. p. 1-21.

30. Hartman TJ, Albanes D, Rautalahti M, et al. Physical activity and prostate cancer in the alpha-tocopherol, beta-carotene (atbc) cancer prevention study (finland). Cancer Causes Control. 1998;9(1):11-18.

31. Johnsen NF, Tjønneland A, Thomsen B, et al. Physical activity and risk of prostate cancer in the european prospective investigation into cancer and nutrition (epic) cohort. Int J Cancer. 2009;125(4):902-908.

32. Nilsen TL, Johnsen R, Vatten L. Socio-economic and lifestyle factors associated with the risk of prostate cancer. Br J Cancer. 2000;82(7):13581363.

33. Putnam SD, Cerhan JR, Parker AS, et al. Lifestyle and anthropometric risk factors for prostate cancer in a cohort of iowa men. Ann Epidemiol. 2000;10(6):361-369.

34. Zeegers MP, Dirx MJ, van den Brandt PA. Physical activity and the risk of prostate cancer in the netherlands cohort study, results after 9.3 years of follow-up. Cancer Epidemiol Biomarkers Prev. 2005;14:1490-1495.

35. Grotta A, Bottai M, Adami H-O. Physical activity and body mass index as predictors of prostate cancer risk. World J Nephrol Urol. 2015;33:14951502 .

36. Hrafnkelsdóttir SM, Torfadóttir JE, Aspelund T, et al. Physical activity from early adulthood and risk of prostate cancer: A 24-year follow-up study among icelandic men. Cancer Prev Res. 2015;8(10):905-911.

37. Clarke G, Whittemore AS. Prostate cancer risk in relation to anthropometry and physical activity: The national health and nutrition examination survey I epidemiological follow-up study. Cancer Epidemiol Biomarkers Prev. 2000;9(9):875-881.

38. Norman A, Moradi T, Gridley G, et al. Occupational physical activity and risk for prostate cancer in a nationwide cohort study in sweden. $\mathrm{BrJ}$ Cancer. 2002;86(1):70-75.

39. Orsini N, Bellocco R, Bottai M, et al. A prospective study of lifetime physical activity and prostate cancer incidence and mortality. $\mathrm{Br} J$ Cancer. 2009;101(11):1932-1938.

40. Parent MÉ, Rousseau MC, El-Zein M, et al. Occupational and recreational physical activity during adult life and the risk of cancer among men. Cancer Epidemiol. 2011;35(2):151-159

41. Vidarsdottir H, Gunnarsdottir HK, Olafsdottir EJ, et al. Cancer risk by education in iceland; a census-based cohort study. Acta Oncologica. 2008;47(3):385-390.

42. Doolan GW, Benke G, Giles GG, et al. A case control study investigating the effects of levels of physical activity at work as a risk factor for prostate cancer. Environ Health. 2014;13:64.

43. Hosseini M, Seyed ASA, Mahmoudi M, et al. A case-control study of risk factors for prostate cancer in iran. Acta medica Iranica. 2010;48(1):6166.

44. Lacey Jr JV, Deng J, Dosemeci M, et al. Prostate cancer, benign prostatic hyperplasia and physical activity in Shanghai, China. Int J Epidemiol. 2001;30(2):341-349.

45. Sass-Kortsak AM, Purdham JT, Kreiger N, et al. Occupational risk factors for prostate cancer. Am J Ind Med. 2007;50(8):568-576.
46. Friedenreich C, McGregor S, Courneya K, et al. Case-control study of lifetime total physical activity and prostate cancer risk. Am J Epidemiol. 2004;159(8):740-749.

47. Lagiou A, Samoli E, Georgila C, et al. Occupational physical activity in relation with prostate cancer and benign prostatic hyperplasia. Eur $J$ Cancer Prev. 2008;17(4):336-339.

48. Wiklund F, Lageros YT, Chang E, et al. Lifetime total physical activity and prostate cancer risk: A population-based case-control study in sweden. Eur J Epidemiol. 2008;23(11):739-746.

49. Bairati I, Larouche R, Meyer F, et al. Lifetime occupational physical activity and incidental prostate cancer (canada). Cancer Causes Control. 2000;11(8):759-764.

50. Krishnadasan A, Kennedy N, Zhao Y, et al. Nested case-control study of occupational physical activity and prostate cancer among workers using a job exposure matrix. Cancer Causes Control. 2008;19(1):107-114.

51. Pierotti B, Altieri A, Talamini R, et al. Lifetime physical activity and prostate cancer risk. Int J Cancer. 2005;114(4):639-642.

52. Strom SS, Yamamura Y, Flores-Sandoval FN, et al. Prostate cancer in Mexican Americans: Identification of risk factors. The prostate. 2008;68(5):563-570.

53. Villeneuve PJ, Johnson KC, Kreiger N, et al. Risk factors for prostate cancer: Results from the canadian national enhanced cancer surveillance system. Cancer Causes Control. 1999;10(5):355-367.

54. Crespo CJ, Garcia-Palmieri MR, Smit E, et al. Physical activity and prostate cancer mortality in puerto rican men. $J$ Phys Act Health. 2008;5(6):918-929.

55. Giovannucci E, Leitzmann M, Spiegelman D, et al. A prospective study of physical activity and prostate cancer in male health professionals. Cancer Res. 1998;58(22):5117-5122.

56. Lee IM, Sesso HD, Paffenbarger RS. A prospective cohort study of physical activity and body size in relation to prostate cancer risk (United States). Cancer Causes Control. 2001;12(2):187-193.

57. Littman AJ, Kristal AR, White E. Recreational physical activity and prostate cancer risk (United States). Cancer Causes Control. 2006;17(6):831-841.

58. Liu S, Lee I-M, Linson P, et al. A prospective study of physical activity and risk of prostate cancer in us physicians. Int J Epidemiol. 2000;29(1):2935 .

59. Platz EA, Leitzmann MF, Michaud DS, et al. Interrelation of energy intake, body size, and physical activity with prostate cancer in a large prospective cohort study. Cancer res. 2003;63(23):8542-8548.

60. Giovannucci EL, Liu Y, Leitzmann MF, et al. A prospective study of physical activity and incident and fatal prostate cancer. Arch Intern. 2005;165(9):1005-1010.

61. Moore SC, Peters TM, Ahn J, et al. Physical activity in relation to total, advanced, and fatal prostate cancer. Cancer Epidemiol Biomarkers Prev. 2008;17(9):2458-2466.

62. Nilsen TI, Romundstad PR, Vatten LJ. Recreational physical activity and risk of prostate cancer: A prospective population-based study in norway (the hunt study). Int J Cancer. 2006;119(12):2943-2947.

63. Patel AV, Rodriguez C, Jacobs EJ, Solomon L, Thun MJ, Calle EE. Recreational physical activity and risk of prostate cancer in a large cohort of us men. Cancer Epidemiol Biomarkers Prev. 2005;14(1):275-279. 
64. Wannamethee S, Shaper A, Walker M. Physical activity and risk of cancer in middle-aged men. Br J Cancer. 2001;85(9):1311-1316.

65. Chen Y, Chiang C, Lin R, et al. Diet, vegetarian food and prostate carcinoma among men in taiwan. Br J Cancer. 2005;93(9):1057-1061.

66. Sanderson M, Coker AL, Logan P, et al. Lifestyle and prostate cancer among older african-american and caucasian men in south carolina. Cancer Causes Control. 2004;15(7):647-655.

67. Darlington GA, Kreiger N, Lightfoot N, et al. Prostate cancer risk and diet, recreational physical activity and cigarette smoking. Chronic Dis Can. 2007;27(4):145-153.

68. Jian L, Shen ZJ, Lee AH, et al. Moderate physical activity and prostate cancer risk: A case-control study in china. Eur J Epidemiol. 2005;20(2):155-160.

69. Yu H, Harris RE, Wynder EL. Case-control study of prostate cancer and socioeconomic factors. The Prostate. 1988;13(4):317-325.

70. Merrill RM, Perego UA, Heiner SW. Age, lifestyle, health risk indicators, and prostate-specific antigen scores in men participating in the world senior games. Rol Oncol-Semin Ori. 2002;105-109.

71. Oliveria SA, Trichopoulos D, Blair S. The association between cardiorespiratory fitness and prostate cancer. Med Sci Sports Exerc. 1996;28(1):97-104.

72. Hållmarker U, James S, Michaëlsson K, et al. Cancer incidence in participants in a long-distance ski race (vasaloppet, sweden) compared to the background population. Eur J Cancer. 2015;51(4):558-568.

73. Galvão DA, Taaffe DR, Spry N, et al. Exercise preserves physical function in prostate cancer patients with bone metastases. Med Sci Sports Exerc. 2018;50(3):393-399.

74. Richman EL, Kenfield SA, Stampfer MJ, et al. Physical activity after diagnosis and risk of prostate cancer progression: data from the cancer of the prostate strategic urologic research endeavor. Cancer Res. 2011;71(11):3889-3895.

75. Barb D, Williams CJ, Neuwirth AK, et al. Adiponectin in relation to malignancies: a review of existing basic research and clinical evidence. Am J Clin Nutr. 2007;86(3):S858-S866.
76. Haverkamp J, Charbonneau B, Ratliff TL. Prostate inflammation and its potential impact on prostate cancer: a current review. J Cell Biochem. 2008;103(5):1344-1353.

77. Nicklas BJ, Hsu FC, Brinkley TJ, et al. Exercise training and plasma C-reactive protein and interleukin-6 in elderly people. J Am Geriatr Soc. 2008;56(11):2045-2052.

78. McCullough DJ, Stabley JN, Siemann DW, et al. Modulation of blood flow, hypoxia, and vascular function in orthotopic prostate tumors during exercise. J Natl Cancer Inst. 2014;106(4):dju036.

79. Lehrer S, Diamond EJ, Stagger S, et al. Increased serum insulin associated with increased risk of prostate cancer recurrence. Prostate. 2002;50(1):13.

80. Roddam AW, Allen NE, Appleby P, et al. Insulin-like growth factors, their binding proteins, and prostate cancer risk: analysis of individual patient data from 12 prospective studies. Ann Intern Med. 2008;149(7):461-71.

81. Leung PS, Aronson WJ, Ngo TH, et al. Exercise alters the IGF axis in vivo and increases p53 protein in prostate tumor cells in vitro. $J$ Appl Physiol. 2004;96(2):450-454.

82. Gardner JR, Livingston PM, Fraser SF. Effects of exercise on treatmentrelated adverse effects for patients with prostate cancer receiving androgen-deprivation therapy: a systematic review. $J$ Clin Oncol Off J Am Soc Clin Oncol. 2014;32(4):335- 346.

83. Hydock DS, Wonders KY, Schneider CM, et al. Androgen deprivation therapy and cardiac function: effects of endurance training. Prostate Cancer Prostatic Dis. 2006;9:392-398.

84. Severson RK, Nomura AM, Grove J S, et al. A prospective analysis of physical activity and cancer. Am J Epidemiol. 1989;130(3):522-529.

85. Robertson JD, Maughan RJ, Duthie GG, et al. Increased blood antioxidant systems of runners in response to training load. Clin Sci. 1991;80(6):611618. 